\title{
Observations of the galvanostatic dissolution of pure magnesium
}

\author{
N.T. Kirkland ${ }^{\mathrm{a}, \mathrm{b}}$, G. Williams ${ }^{\mathrm{c}}$, N. Birbilis ${ }^{\mathrm{a}, *}$ \\ ${ }^{a}$ Department of Materials Engineering, Monash University, Clayton, Victoria, 3800. Australia \\ bepartment of Advanced Technology and Science for Sustainable Development, Nagasaki \\ University, Nagasaki, Japan \\ ${ }^{\mathrm{b}}$ Materials Research Centre, College of Engineering, Swansea University, Singleton Park, Swansea. \\ SA2 8PP, Wales. UK \\ * Tel: +61-3990-54919, Fax: +61-9905-4940, \\ Email: nick.birbilis@monash.edu
}

\begin{abstract}
:
The dissolution of pure magnesium in $0.1 \mathrm{M} \mathrm{NaCl}$ under galvanostatic polarisation is reconciled in real time via video imaging of specially designed working electrodes that permit geometrically uniform dissolution. The dissolution stoichiometry was observed to be centred about $n=2$, however this varies according to the magnitude of the applied current. Applied currents densities $<1 \mathrm{~mA} / \mathrm{cm}^{2}$ display $n \approx 1.75-2$, whilst densities $>1 \mathrm{~mA} / \mathrm{cm}^{2}$ display $\mathrm{n} \geq 2$, the latter related to a significant increase in electrolyte $\mathrm{pH}$ during testing.
\end{abstract}

Keywords: A: magnesium, B: galvanostatic, C: anodic dissolution. 
The poor corrosion resistance of magnesium $(\mathrm{Mg})$ and its alloys continues to be a major barrier to industrial uptake of $\mathrm{Mg}$ as a structural material. Consequently, unambiguously defining the dissolution characteristics of $\mathrm{Mg}$ remains an important research topic. In spite of the practical importance, there are both incomplete and conflicting perspectives in the literature in regards to the mechanisms of Mg dissolution in aqueous electrolytes [1-10]. In order to fully quantify the dissolution kinetics, a number of analytical techniques would be required in a full dedicated study, which is not the aim of this work, whereby we aim to correlate the dissolution expected from an electrochemical (anodic) signal, and the observed dissolution in real time. More specifically, the purpose of the work herein is to add to independent works with a view to correlation of electrochemistry with metal loss, providing a ground truth result as opposed to implied correlations from separate tests. The correlation between galvanostatically applied signals and metal loss is fundamentally given by Faraday's law that defines the relationships between the mass of a metal and the electrical charge required to alter it, shown in Eqn. 1:

$$
m=\left(\frac{Q}{F}\right)\left(\frac{M}{n}\right) \quad \text { Eqn. } 1
$$

where $m$ is the mass of the substance liberated at an electrode (in g), $Q$ is the total electric charge passed through the substance (C), $F$ is Faraday's constant $\left(96,485 \mathrm{C} \mathrm{mol}^{-1}\right), M$ is the molar mass of the substance $\left(\mathrm{g} \mathrm{mol}^{-1}\right.$ ), and $n$ is the valency number of ions of the substance (electrons transferred per ion). For pure Mg, these values are; $M=24.31 \mathrm{~g} \mathrm{~mol}^{-1}$ and $n=2$. $Q$ can be simply defined as the current applied $(I)$ by the time $(t)$ over which this current is applied $(I t=Q)$.

In the 1950s Petty [6] was the first to suggest that Mg may be ionised with a stoichiometry not equal to 2 , but $n=1$. The evidence given in that work was rather limited in terms of raw data, however several decades later the notion of the unipositive $\mathrm{Mg}^{+}$continues to be used as a description in some studies [10], suggested by some to explain why the quantity of Mg that is found to experimentally dissolve is often greater than explained by Faraday's law. In such studies however, the observations are nominally based on inferred correlations that involved comparison of information from hydrogen collection experiments versus polarisation experiments or even mass loss (i.e. long term versus short- 
term tests which may be influenced by time dependencies such as electrolyte variation). Such theories revolve around the idea that unipositive $\mathrm{Mg}^{+}$is involved as an early step in the electrochemical oxidation of magnesium and moreover that the unipositive $\mathrm{Mg}^{+}$has a sufficient lifetime to react chemically with other available species [1-4], Such a process is frequently termed “anodic" hydrogen evolution since proposed the $\mathrm{Mg}^{+}$reductant is the product of an anodic process. It is prudent to point out that recent works have shown (by independent techniques) that Mg dissolves with a stoichiometry closer to $n=2$. This includes works using SVET derived current density distributions (analysed to yield local current) [8]. Additionally, a novel experimental arrangement called atomic emission spectroelectrochemistry, which includes in-line optical emission spectrometry, has also shown rather vividly that Mg dissolves with a stoichiometry of $n=2$ under an applied anodic electrochemical signal [5]. Such independent works are in direct conflict with the notions of unipositive $\mathrm{Mg}^{+}$, including works that have defined the "Negative Difference Effect" [1, 3-4, 7]. It has been posited [7] that the more $\mathrm{Mg}$ ions that enter the solution, the stronger the water dissociation and more electrons are supposedly consumed by hydrogen ions - however physical evidence for this is not complete.

In this work, ultra high purity Mg (99.99\%, Timmenco, Toronto, Canada) with <10ppm Fe was cut into a flat triangular wedge specimens (dimensions in Figure 1A). This shape, and thinness, was designed such that dissolution would not lead to samples prematurely failing from portions falling off non-faradaically. Prior to testing, samples were ground to 2000 grit, an insulated copper wire was attached to the bottom (blue) portion of the sample, and air-curing silicone sealant was used to seal the wire and sample to a glass slide. Care was taken to ensure that only the triangular area (green) was exposed. The exposed area had a total volume of $0.0105 \mathrm{~cm}^{3}$. This assembly was tested thoroughly for robustness and reproducibility - reported results being repeated at least in triplicate. The sample was placed in a Pyrex ${ }^{\circledR}$ corrosion cell which contained a LIDA $^{\text {TM }}$ mixed metal oxide coated titanium mesh counter electrode (comprised of pure Ti mesh with an applied coating of platinum group metal oxides). $200 \mathrm{ml}$ of $0.1 \mathrm{M} \mathrm{NaCl}$ was used as the electrolyte. The video recording setup consisted of a ToupTek 3.1 megapixel USB microscope camera (Hangzhou ToupTek Photonics Co., China) capturing images every 60 seconds and attached to an Olympus SZ40 stereo microscope, placed 
horizontally. A ring-light was used to provide even lighting to the entire sample (Figure1B). Samples were polarised using a BioLogic ${ }^{\circledR}$ VMP 3 potentiostat under control of EC-Lab 10.17 software (BioLogic Inc., TN, USA). In addition to open circuit corrosion, applied galvanostatic currents of 0.5, 1, 2, 5, 10, and $20 \mathrm{~mA}$ were investigated (the corresponding initial current density values given in Table 1). To give the reader an appreciation of the potentials achieved during such experiments, a galvanodynamic polarisation curve is presented (Figure 2), collected using a conventional 3-electrode flat cell (PAR®).

Some raw images of the specimens are included in Figure 3. These images correspond to various times in the total test time for the given samples, and reveal the typical features that can be detected from in-situ observation of dissolution in real time. In all cases, videos are also supplied for a typical test run at each of: open circuit [11], a low [12] medium [13] and high [14] applied current density. The videos form an integral part of the data in the paper, and are thus included as supplementary information also (noting that the links in the references provide high resolution videos, with the supplementary files being regular resolution). What is immediately obvious from the videos is that the dissolution can be tracked rather accurately, and that there are differences between the dissolution characteristics (namely time) as a function of applied current. The videos have been adjusted to be $\sim 2$ mins in length with the corresponding time code given with each filename. We also note that galvanostatic experiments, the actual current density applied is a function of the specimen size, and this is reconciled in Figure 4. It is also seen in Figure 4 that 'end point' analysis was executed when $90 \%$ of the specimen was dissolved, after which the current density increases asymptotically. One obervation that is immediately obvious is that in spite of the specimens being polarised anodically (which implies the cathodic reaction occurs on the counter electrode), there was evidence of hydrogen bubbles emanating from the anode surface. The source of hydrogen evolution under anodic polarisation of $\mathrm{Mg}$ is a major point of debate, with many publications [1-4] proposing the existence of $\mathrm{Mg}^{+}$as an explanation of its occurrence, as discussed earlier. However, an alternative postulation is that the hydrogen derives from a cathodic reaction which persists even under anodic polarisation, and that the net current flowing at the Mg anode is the sum total of the anodic current $\left(\mathrm{I}_{\mathrm{a}}\right)$ and a non-zero 
cathodic current $\left(I_{c}\right)$, even well above the open circuit potential. The work herein aims to use the observations of galvanostatically polarised Mg specimens to clarify which of the above explanations may be correct.

The videos presented, more accurately the stills from the videos, can be readily analysed using digital image analysis to yield the percentage dissolved (i.e. volume loss). Results from such analysis are presented in an abridged form in Figure 5.

The charge required to dissolve the specimen of known volume (to an end point of $90 \%$ dissolved as depicted in Fig. 3) is plotted versus the applied current (Fig. 5A, and with log-axes in Fig. 5B). Since the evolving specimen geometry leads to an increasing current density with test duration - this is accounted for in Figure 5C - whereby the time to dissolution versus theoretical time to dissolution is presented. Analysis of the difference between the theoretical and actual time to dissolution displays two interesting phenomena (Fig. 5D). At the lower end of applied currents, the specimens dissolved faster than predicted (initial applied current density of $<1 \mathrm{~mA} / \mathrm{cm}^{2}$ ) whereas for an initial applied current density of $>1 \mathrm{~mA} / \mathrm{cm}^{2}$, the time to dissolution was increasingly longer than that predicted from Faraday's law (for comparison, the time to dissolution from open circuit (un-polarised) exposure is also included in Fig. 5C).

In the analysis of data, Figure 3A immediately reveals that the charge required to dissolve the specimens is not identical for the various applied currents. Overlaid lines indicate the charge required for a classical $n=2$ stoichiometry, along with an $n=1$ (unipositive Mg), included simply for revealing the scatter in the measured results. The lowest current tested (Table 1) reveals that the charge required to cause specimen dissolution was consistent with a stoichiometry of $\sim n=1.75$. This increased to a precise $n=2$ as applied current increased to $2 \mathrm{~mA}$, whilst the charge requirement exceeded any theoretical predictions for further increases in current. The latter situation is one that is not often reported, but a salient feature of many long-term corrosion tests upon Mg whereby the result may be influenced by alteration of electrolyte chemistry (specifically $\mathrm{pH}$ ). To reveal this effect, we also provide videos for open circuit [15], 0.5mA [16] and $20 \mathrm{~mA}$ [17] dissolution in the presence of 
$\mathrm{pH}$ indicator (of which $1 \mathrm{~mL}$ was added to the electrolyte). The indicator tends towards purple at alkaline $\mathrm{pH}$ (transitioning via pink hues when $\mathrm{pH}$ exceeds neutral). The results clearly reveal that electrolyte alkalisation occurs in all cases (including open circuit). It is however not possible from such tests to isolate whether the ultimate alkalisation is from excessive surface alkalisation of $\mathrm{Mg}$, or from diffusion from the counter electrode - none the less, the electrolyte $\mathrm{pH}$ alteration will allow for surface hydroxides $\left(\mathrm{Mg}^{2+}+2 \mathrm{OH}^{-} \Rightarrow \mathrm{Mg}(\mathrm{OH})_{2}\right)$ to form, stimulating some passivity, and excess charge needed to ultimately cause dissolution. In the case of $20 \mathrm{~mA}$ applied current, the charge required to cause dissolution almost twice that required at $0.5 \mathrm{~mA}$ - which is an important finding in itself. Overall, a summary of results is given in Table 1, and readers are also capable of performing any of their own analysis via the raw data available [11-14].

In regards to the results that suggest a stoichiometry somewhat below $n=2$, a number of factors were considered. Firstly, it was considered that non-Faradaic dissolution may have occurred (i.e. grain fallout or undermining of portions of specimen), however repeat tests and the relative accuracy of image analysis gave confidence that such factors were not dominant. The potential of the presence of chloride ions to cause erractic disolution was also considered. In the work of Swiatowska et al. [5], it was observed that a much closer relation ship to $n=2$ was realised when testing in $\mathrm{Cl}^{-}$free electrolyte, such as $\mathrm{Na}_{2} \mathrm{SO}_{4}$. As such, it was envisaged to replicate all data collection in $10 \mathrm{mM} \mathrm{Na}_{2} \mathrm{SO}_{4}$, however this was abandoned since the dissolution response of $\mathrm{Mg}$ in $\mathrm{Na}_{2} \mathrm{SO}_{4}$ resulted in the accumulation of insoluble product that frustrated image analysis - also noted in [5]. Clear evidence of this is seen in the corresponding videos [18-19], which show enough contrast to reveal metal dissolution inwards but sample geometry retention in the form of a white corrosion product. In another video, [20] we show that poking the corrosion product leads to its disintegration, hence confirming that complete dissolution of Mg metal had occurred. What can be inferred from the work herein is that if Mg did dissolve via an $n=1$ stoichiometry, then such a notion, combined with "anodic" hydrogen evolution, ought to have led to an obviously lower charge required for dissolution - which was not observed. Additionally, the Negative Difference Effect that is defined as an increase in hydrogen evolution with an increase in anodic current density is in conflict with the observations herein. The videos presented- 
which are the first of their kind - do not show increased hydrogen evolution upon the anode with increasing anodic current based on qualitative observation. In the absence of being able to collect hydrogen specifically from the anode in a polarised test whilst simultaneously video recording, only a qualitative assessment is possible herein, however future work will seek to simultaneously collect hydrogen in tandem with quartz crystal microbalance studies. A purely visual assessment suggests the largest amount of hydrogen evolved is observed at open circuit [11-14], whereby increasing anodic current, the prevalence of local cathodes diminished.

In summary, these results show that $\mathrm{Mg}$ dissolution occurs with an apparently different stoichiometry for the varying conditions of these experiments. It appears that a slightly less than $n=2$ stoichiometry was observed for the lowest current density tested, relating with a slightly higher practical dissolution rate - but not sufficient for $n=1$. We also note that under anodic galvanostatic polarisation local cathodes exist, to a varying extent, even when an anodic current $\left(I_{a}\right)$ is imposed (qualitatively judged from the evidence of evolved hydrogen). Consequently the simple assumptions that applied anodic current $I_{a, a p p l}=I_{a}$ are not correct, since a cathodic current, $I_{c}$, exists even under anodic polarisation (however it is conceded that quantification of the cathodic current would require specific testing such as SVET, which is not done herein). This observation in itself would tend to invalidate assertions for the unipositive $\mathrm{Mg}^{+}$that are implied on the basis of $\mathrm{I}_{\mathrm{a}, \mathrm{appl}}=\mathrm{I}_{\mathrm{a}}$. Furthermore, it also highlights a weakness in the ability of hydrogen collection measurements to serve as an index to dissolution in the case of $\mathrm{Mg}$, and more so on theories of the existence of unipositive $\mathrm{Mg}^{+}$based on comparison of hydrogen collection data with other independently collected data. In the case of a large applied current density, a charge $(\mathrm{Q})$ beyond that expected theoretically (i.e. $n>2$ ) is required to dissolve $\mathrm{Mg}$, most likely owing to the rapid increase in electrolyte $\mathrm{pH}$ that results in the possibility of Mg-hydroxide products to form. The data in Figure 5D reveals an interesting observation where there is an overlap in regime from underpredicting the amount of dissolution occurring (regime (i)), to overpredicting (regime (ii)). This phenomenologically represents a transition from a situation whereby significant anodic hydrogen evolution is occurring, to one where the electrolyte is dynamically altering. It is also finally noted that of the works to show $n=2$ [5,8 and herein], the results have been from direct 
measurements in real time (i.e. SVET, AESEC and video), indicating that drawing parallels from separate - often unrelated (i.e. short term versus long term) tests can be misleading. 


\section{References:}

1. A. Atrens, W. Dietzel, Advanced Engineering Materials, 9 (2007) 292-297.

2. T.R. Thomaz, CR. Weber, T. Pelegrini, L.F.P. Dick, G. Knörnschild, Corrosion Science, 52 (2010) 2235-2243.

3. G. Song, A. Atrens, D. St John, X. Wu, J. Nairn, Corrosion Science 39 (1997) 19812004.

4. G. Song, A. Atrens, D. St John, J. Nairn, Y. Li, Corrosion Science, 39 (1997) 855-875.

5. J. Światowska, P. Volovitch, K. Ogle, Corrosion Science 52 (2010) 2372-2378.

6. R.L. Petty, A.W. Davidson, J. Kleinberg, Journal of the American Chemical Society, 76 (1954) 363-363.

7. S. Bender, J. Goellner, A. Heyn, E. Boese, Materials and Corrosion, 58 (2007) 977-982.

8. G. Williams, H.N. McMurray, Journal of the Electrochemical Society, 155 (7) (2008) C340-349.

9. J. Przyluski, E. Palka, Electrochim. Acta, 15 (1970) 853-864.

10. D. Sachdeva, Corrosion Science 60 (2012) 18-31.

11. http://users.monash.edu.au/ nickbir/OpenCircuit(1s=15mins).wmv

12. http://users.monash.edu.au/ nickbir/05mA(1s=10mins).wmv

13. http://users.monash.edu.au/ nickbir/2mA(1s=5mins).wmv

14. http://users.monash.edu.au/ nickbir/20mA(1s=1min).wmv

15. http://users.monash.edu.au/ nickbir/OpenCircuit_pHTest(1s=5min).wmv

16. http://users.monash.edu.au/ nickbir/05mA_pHTest(1s=60s).wmv

17. http://users.monash.edu.au/ nickbir/20mA_pHTest(1s=60s).wmv

18. http://users.monash.edu.au/ nickbir/20mA_Na2SO4(1s=1min).wmv

19. http://users.monash.edu.au/ nickbir/05mA_Na2SO4(1s=10min).wmv

20. http://users.monash.edu.au/ nickbir/Poking_Products_from_Na2SO4.m4v 
Table 1: Values of time to specimen dissolution and charge required for dissolution from both theory and as recorded. Average values herein, with range of scatter presented by error bars in Figure 3 .

\begin{tabular}{llll}
\hline $\begin{array}{l}\text { Applied current } \\
\text { (mA) }\end{array}$ & $\begin{array}{l}\text { Applied initial } \\
\text { current density } \\
\left(\mathbf{m A} / \mathrm{cm}^{2}\right)\end{array}$ & $\begin{array}{l}\text { Average Actual } \\
\text { dissolution time } \\
\text { (min) }\end{array}$ & $\begin{array}{l}\text { Average Total } \\
\text { charge for } \\
\text { dissolution, Q } \\
\text { (C) }\end{array}$ \\
\hline $\mathbf{2 0}$ & 4.4 & 110 & 199 \\
$\mathbf{1 0}$ & 2.2 & 180 & 180 \\
$\mathbf{5}$ & 1.1 & 240 & 155 \\
$\mathbf{2}$ & 0.45 & 530 & 143 \\
$\mathbf{1}$ & 0.23 & 740 & 134 \\
$\mathbf{0 . 5}$ & 0.12 & 900 & 121 \\
Open circuit & $\mathbf{n} / \mathbf{a}$ & $\mathbf{1 1 5 0}$ & $\mathbf{1 4 5}$ \\
\hline
\end{tabular}

${ }^{*}$ Corresponds to theoretical value for a known specimen volume and $n=2$. 


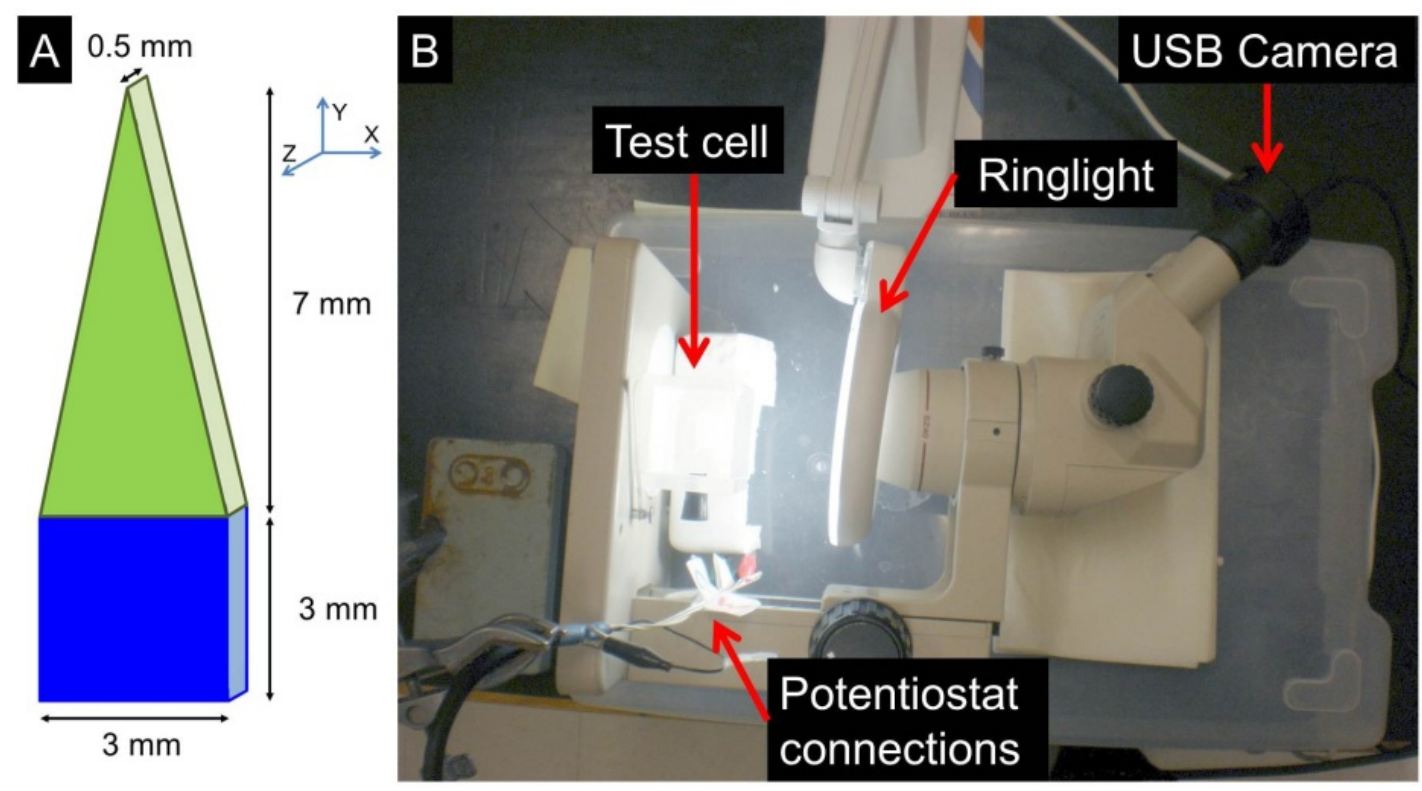

Figure 1: (A) Diagram of Mg sample, where green area indicates surface that was exposed to electrolyte. (B) Photograph of corrosion and recording setup with labels. 


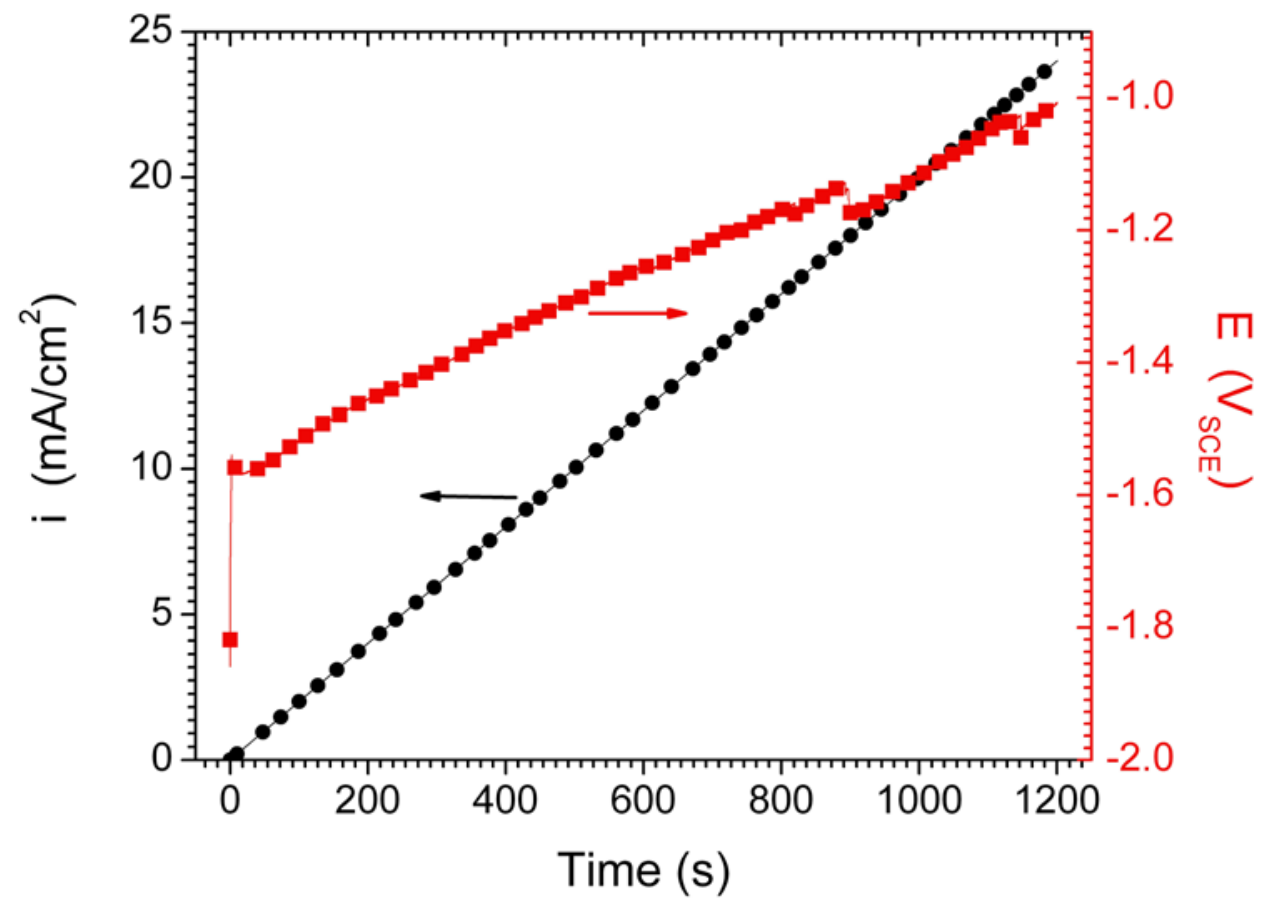

Figure 2: Evolution of potential as a function of increasing current during an anodic galvanodynamic test of pure $\mathrm{Mg}$ performed at a scan rate of $0.02 \mathrm{~mA} / \mathrm{s}$ in $0.1 \mathrm{M} \mathrm{NaCl}$. Specimen was at open circuit for 10 minutes prior to commencement of polarisation. 


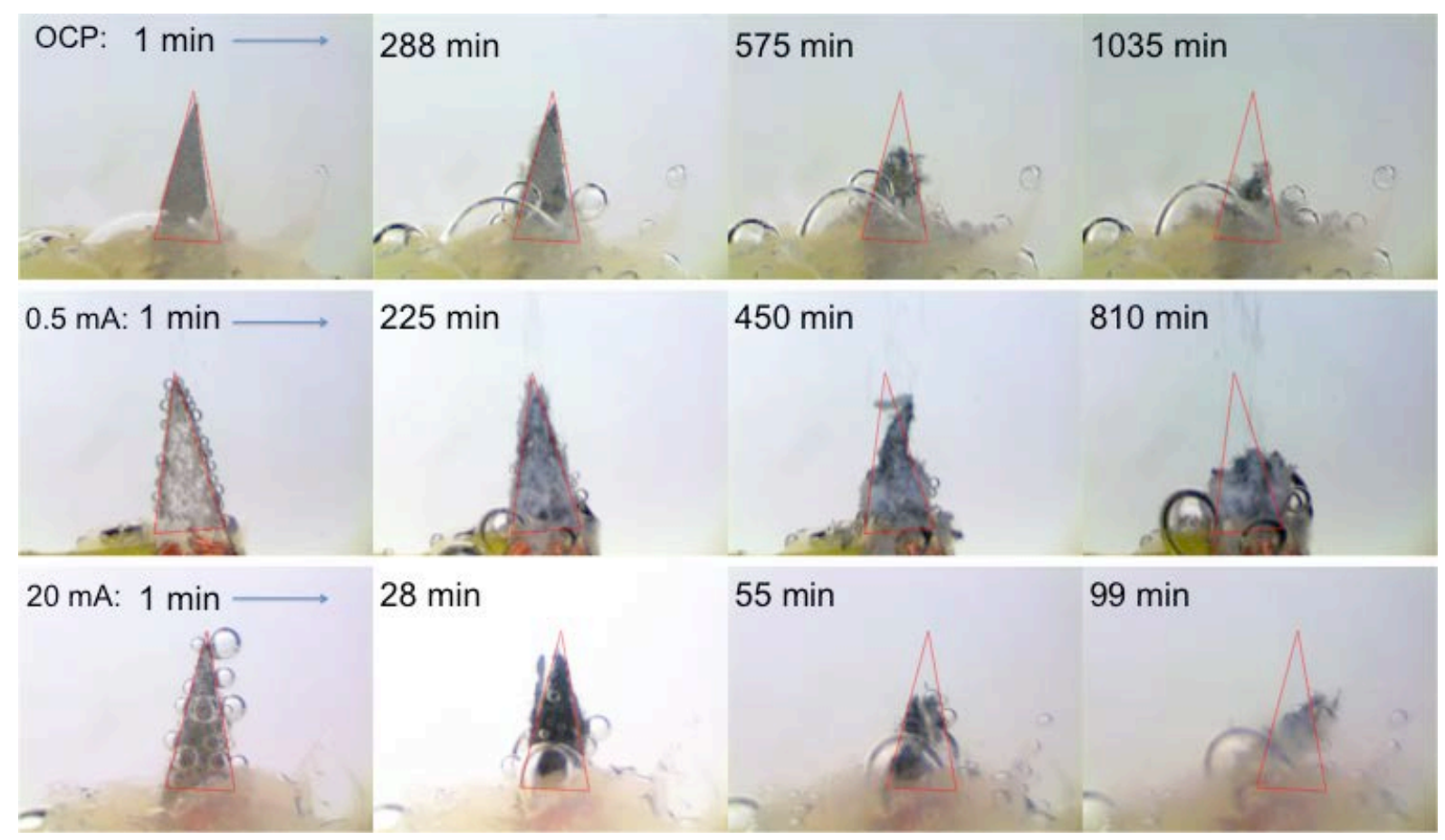

Figure 3: Photographs of sample with no applied current (top), $0.5 \mathrm{~mA}$ (middle) and $20 \mathrm{~mA}$ (bottom) in $0.1 \mathrm{M} \mathrm{NaCl}$. Images represent approximately $0,25,50$, and $90 \%$ of total specimen dissolution.

Outline of original specimen geometry overlaid. 


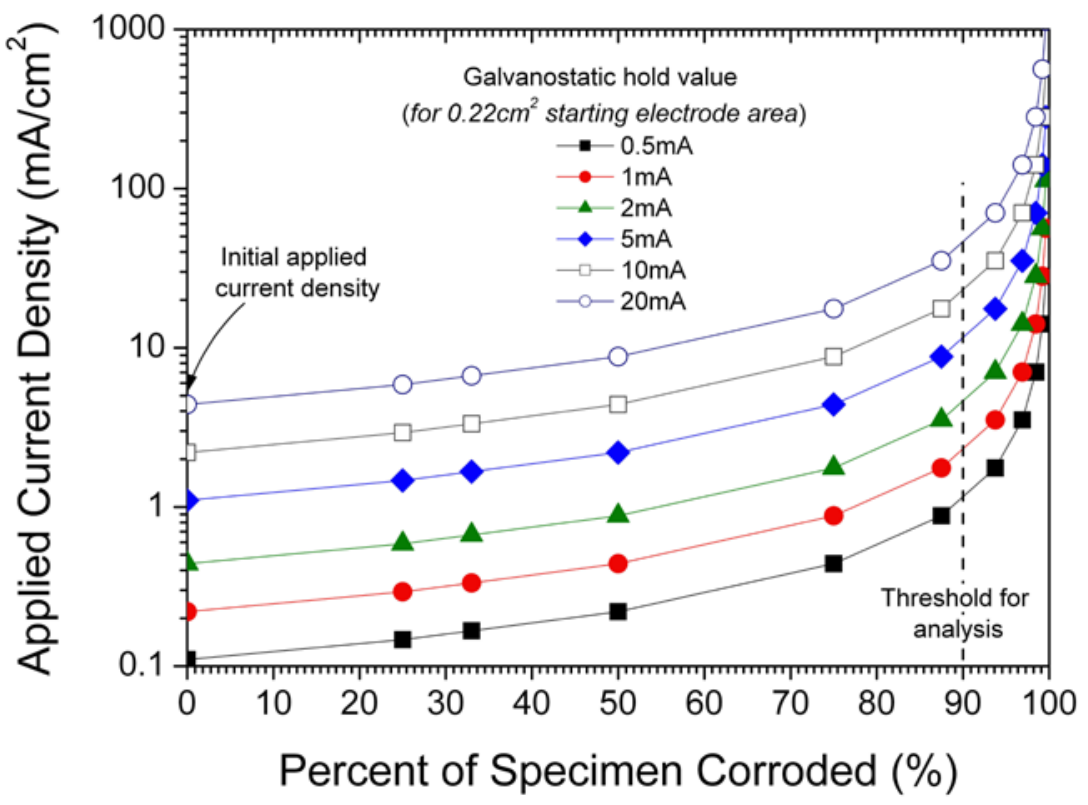

Figure 4: The applied current density as a function of the percentage of specimen corroded during galvanostatic polarisation at $0.5,1,2,5,10$ and 20mA. The analysis 'end point' is noted, prior to the asymptoting of current density (whereby current density doubles each time the specimen loses 50\% of its area). 


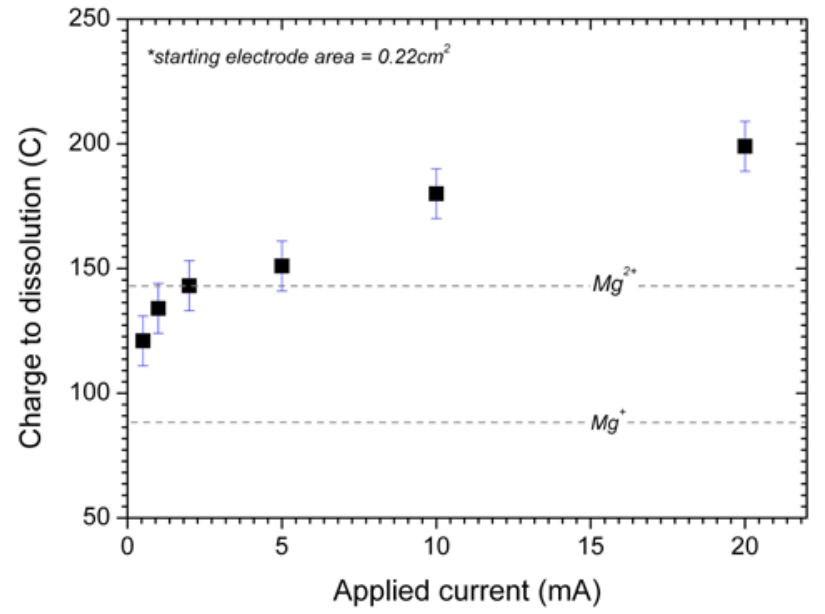

(A)

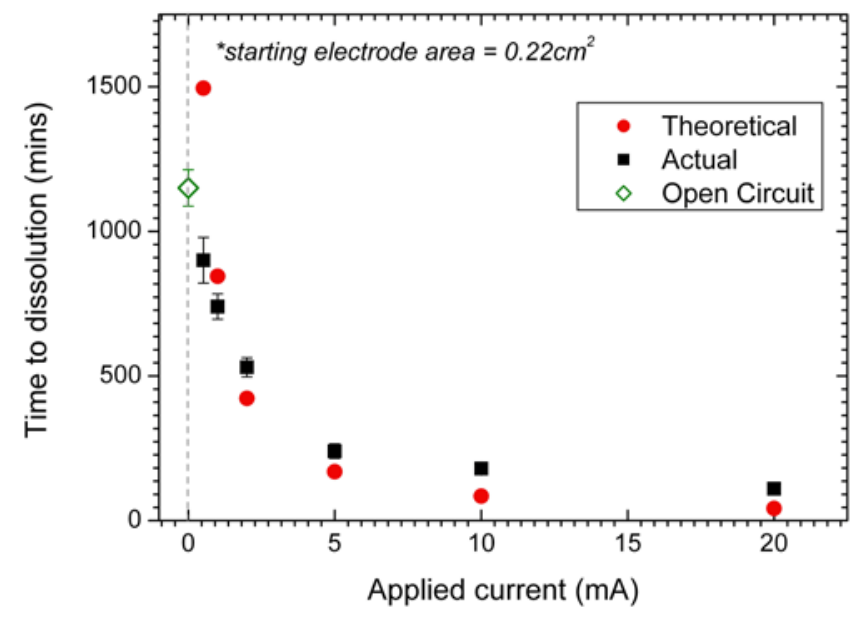

(C)

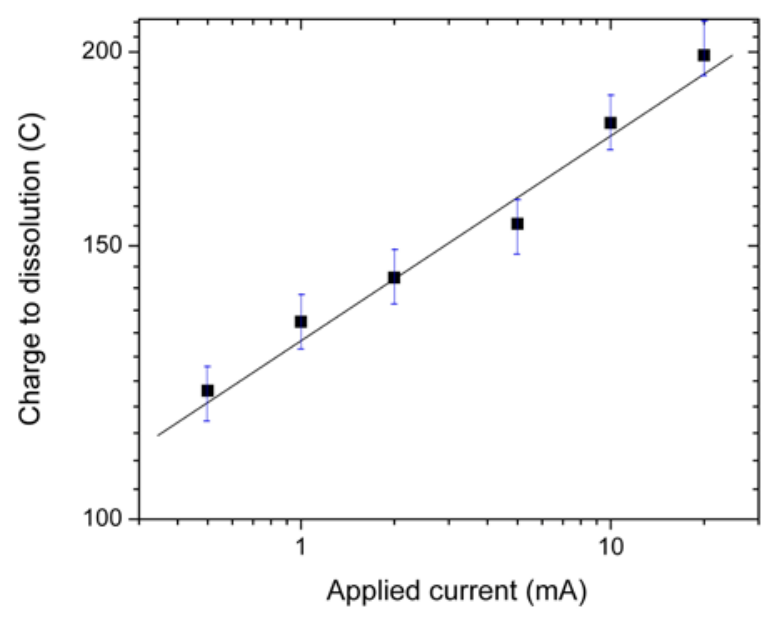

(B)

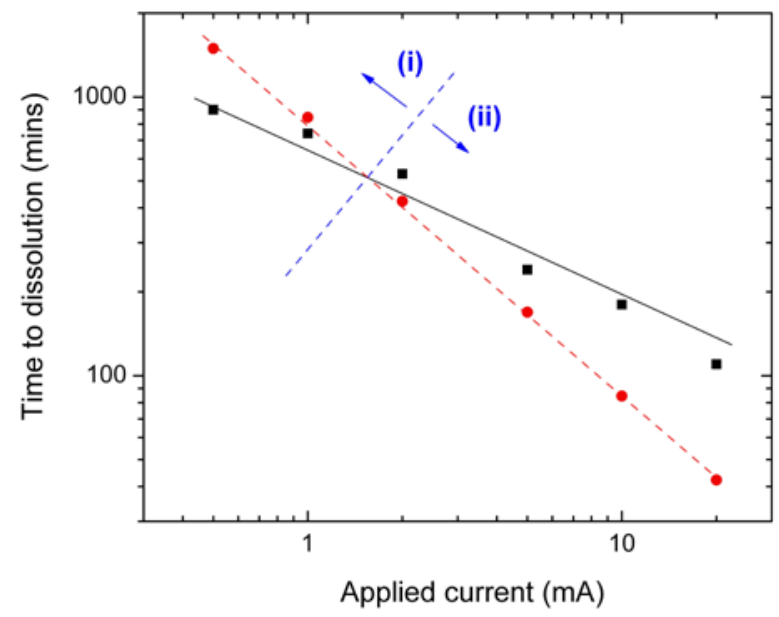

(D)

Figure 5: For various varied applied currents upon pure $\mathrm{Mg}$ in $0.1 \mathrm{M} \mathrm{NaCl}$ : (A) Total charge required for dissolution (with theoretical $\mathrm{Mg}^{+} / \mathrm{Mg}^{2+}$ stoichiometry overlaid); (B) As in A, with double log axes; (C) The actual versus theoretical time (based upon the average actual value) to dissolution for $n=2$, inset reveals log scales; (D) As in C, with double log axes (and error bars have been omitted for clarity) 